\title{
FUNKTIONSWANDEL DEIKTISCHER STÄMME IM NGANASANISCHEN: GRAMMATIKALISIERUNG, LEXIKALISIERUNG, PRAGMATIKALISIERUNG
}

\author{
RÉKA ZAYZON
}

\begin{abstract}
Auszug
Gegenstand der folgenden Arbeit ist die historische Entwicklung der nganasanischen Demonstrativa.

Bekanntlich unterliegt die Kategorie der Demonstrativa (der Raum-, Zeit-, und Objektdeixeis) spezifischen Veränderungen, die durch ihre innere (funktional-semantische) und äussere (syntaktische) Struktur zu erklären sind. Schon seit den Anfängen der historischen Sprachwissenschaft hat man die besondere Rolle der pronominalen Kategorie insgesamt und insbesondere der Demonstrativa bei der Entstehung der primären Kasussuffixe erkannt, im Laufe einer langjährigen Forschungsgeschichte diente das Prinzip als Grundlage zahlreicher Rekonstruktionen. Eine systematische Zusammenfassung typischer Entwicklungslinien (Grammatikalisierungsketten) ist letztlich im Rahmen der Grammatikalisierungsforschung unternommen worden.

Die Zielsetzungen dieses Beitrags ist zweierlei: Einerseits werden Erkenntnisse der Grammatikalisierungsforschung auf das Nganasanische angewandt. (Dabei dient das Nganasanische weniger als Musterbeispiel für verschiedene Grammatikalisierungsketten, eher als mögliches Anwendungsfeld.) Als Ergebnis wird die Entwicklungslinie Ppron3Sg siti > Demonstrativ falsifiziert und einige zusätzliche Entwicklungslinien postuliert, so z. B. die der modalen Verwendungen der Demonstrativa, die in der uralistischen Literatur bislang wenig Beachtung fanden. Ferner werden terminologische Inkonsequenzen der Fachliteratur bei der Beschreibung einzelner Entwicklungslinien als Grammatikalisierung aufgezeigt.
\end{abstract}

1. Deiktische Morpheme waren schon seit den Anfängen der historischen Sprachwissenschaft Gegenstand des Forschungsinteresses und lieferten Musterbeispiele für das Phänomen, das später als Grammatikalisierung bezeichnet wurde. Auch in der seit einigen Jahrzehnten blühenden Grammatikalisierungsforschung spielt Deixis eine zentrale Rolle: Das Thema fehlt in keiner allgemeinen Darstellung, denn sie gilt als eine Kategorie, die auf idiosynkratische Weise grammatikalisiert wird und mehrfache Grammatikalisierungsketten aufweist. Da deiktische Elemente nahezu immer am Anfang eines Grammatikalisierungsprozesses stehen, ${ }^{1}$ gelten sie

${ }^{1}$ Die herrschende Annahme, dass deiktische Elemente immer am Anfang der Grammatikalisierungskette stehen, wurde von Frajzyngier (1996) widerlegt. Er führte 
als Sprachgenetisch ursprünglich und tauchen bezeichnenderweise in Spekulationen über den Ursprung der Sprache auf.

1.1. Gegenstand der folgenden, auf empirischem Sprachmaterial beruhenden Beschreibung sind Verwendungsweisen und die ihnen entsprechenden Funktionen von ursprünglich deiktischen Stämmen sowie deren Zusammensetzungen und Ableitungen in der nganasanischen Sprache. ${ }^{2}$ Die Beschreibung dient dem Ziel, das funktionale und formale Potential ${ }^{3}$ der deiktischen Stämme, die die vielfältigen Funktionswandeln ermöglichen, aufzudecken, und aufzuzeigen, inwieweit diese Funktionswandeln mit der Begrifflichkeit der Grammatikalisierung zu erklären sind.

1.2. Die Abgrenzung der zu beschreibenden Phänomene erfolgt durch Ermittlung etymologisch zusammenhängender Lemmata in Wörterbüchern und Lexika. Ferner werden in der typologischen und uralistischen Fachliteratur bereits beschriebene Grammatikalisierungsketten sowie etymologische Fachliteratur herangezogen. Die Beschreibung beruht auf in der Samojedologie vereinzelt auffindbaren einschlägigen Ausführungen, die nach Möglichkeit ergänzt werden. Nach einer kritischen Anwendung des Begriffs Grammatikalisierung (1.3) wird der Kreis der durch ihn bezeichneten Phänomene umrissen. Schließlich werden Eigenheiten des nganasanischen Systems durch einen Vergleich mit den „klassischen“ Grammatikalisierungsketten aufgezeigt.

1.3. Der Begriff Grammatikalisierung bezeichnet einen komplexen Sprachwandel, der sich auf verschiedene sprachliche Ebenen auswirkt. Betroffen ist stets eine sprachliche Einheit der Größe mindestens eines Syntagmas, das in einem spezifischen Kontext von den Sprechern reinterpretiert wird. Dabei entwickelt sich ein Element (ein Lexem oder ein gebundenes Morphem) des reanalysierten Segments zu einem grammatischen Morphem. Dies geht einher mit

Beispiele aus dem Chadischen dafür an, dass auch Raumdeixis sekundär, d.h. hier im Laufe eines Lexikalisierungsprozesses, entstehen könne. Üblich ist jedoch die Entstehungsweise von Personendeixis aus Symbolfeldausdrücken, siehe hierzu Punkt 2.1.1 und Róna-Tas (1976).

${ }^{2}$ Für eine einführende Beschreibung der nganasanischen Sprache s. Helimski (1998), Tereščenko (1979).

${ }^{3}$ Im Sinne von Redder (1990, 3-6).

Acta Linguistica Hungarica 51, 2004 
(a) semantischer Entleerung und Zunahme an grammatischer Bedeutung

(b) zunehmender syntaktischer oder morphologischer Fixierung

(c) Abschwächung oder Kürzung des Lautbildes

des betroffenen Elements.

Mit der Erweiterung der Forschung im Bereich der Grammatikalisierung wurde der Begriff immer weiter präzisiert. Zunächst wurde der Begriff Lexikalisierung hinzugefügt, um solche Sprachwandelphänomene, an deren Ende eine Erweiterung des Lexikons steht, von der Grammatikalisierung abzugrenzen. Es ist leicht einzusehen, dass es bei dem Begriff Grammatikalisierung Grauzonen und unterschiedliche Meinungen allein schon deswegen geben muss, weil auch der Begriff der Grammatik und somit der grammatischen Bedeutung kein diskret abgrenzbares Konzept darstellen. ${ }^{4}$

In der Fachliteratur werden unter dem Begriff der Grammatikalisierung schließlich auch Sprachwandelphänomene beschrieben, die eine Entwicklung hin zu einer pragmatischen Funktion darstellen (z.B. der Diskurspartikeln - Fernandez-Vest 2000), deren Erfassung weder der Grammatik noch dem Lexikon zusteht. Terminologisch konsequent wäre es, solche Entwicklungen nicht als Grammatikalisierung zu bezeichnen.

1.4. Bedingt durch ihre idiosynkratische innere und äußere Struktur unterliegen deiktische Morpheme regelhaftem Wandel. Dies wird bei etymologischen Rekonstruktionen vorausgesetzt, die Regeln des funktionalen Wandels werden jedoch selten explizit formuliert. ${ }^{5}$

Zur systematischen Ausformulierung der entsprechenden Regeln kam es in der Grammatikalisierungsforschung. Die „klassischen“ Grammatikalisierungsketten von Demonstrativa, wie sie bei Lehmann $(1995,55)$ beschrieben werden, wurden im Laufe der Forschung nach und nach ergänzt.

4 ,Speculation of this kind should warn us that our definition of grammar can only be as truthworthy as our apprehension of the notion of grammar. But the study of grammar itself has revealed no clear boundaries to the concept of grammar. Rather, grammatical phenomena have fuzzy borders and are always changing. Grammar itself is the product of linguists' reflection on language, and thus represents a consensus about appropriate objects of inquiry rather than a sharply delineated, definable entity" (Ramat-Hopper 1998, 4).

${ }^{5}$ So findet sich z.B. bei Closs Traugott (2002) kein Hinweis hierauf. 
Die bislang umfassendste mir bekannte Liste, die aus Diessels einschlägiger Monographie stammt (Diessel 1999a), kann ebenfalls durch einige Angaben ergänzt werden. ${ }^{6}$

Im folgenden soll eine Auflistung der in der einschlägigen Forschungsliteratur beschriebenen (im Einzelfall hypothetischen) Grammatikalisierungsketten deiktischer Morpheme stehen: ${ }^{7}$

- Demonstrativpronomen $>$ Personalpronomen $>$ Personalsuffix $>$ determinierendes Suffix;

- Demonstrativpronomen/anaphorisches Pronomen $>$ Personalpronomen 3sg > Relativpronomen/Konnektiv;

- Demonstrativpronomen $>$ Determinans mit abgeschwächt demonstrativem Charakter $>$ Determinans $>$ Artikel $>$ Suffix der nominalen Klasse (Lehmann 1995, 55);

- Demonstrativpronomen $>$ Personalpronomen $>$ Px in determinativen Funktion $>$ weitere Suffixe mit determinativem Charakter (z.B. das Akkusativsuffix, vgl. Mikola (1965), das Prädestinativsuffix vgl. Künnap 1987; Janhunen 1989);

- konkrete Lokalisierung > Possessivrelation (habeo-Konstruktion und Existentialsatz) (Heine 1992)

- > expletive particle (fr. c'est toi que je connais) (Diessel 1999b);

$->$ Satzkonnektor (ebd.);

- > Converb; Verbalpräfix (ebd.);

$->$ Fokus- und Topikmarker (ebd.);

$->$ Lokalkasussuffix;

- > Tempuszeichen (Heine-Kuteva 2002);

- > Ordnungszahl (? fi. ensi, toinen - Rédei 1988-1991, Itkonen-Kulonen 1992-2000);

- > Evidentialsuffix (Willett 1988, 80);

- > Modal- und Diskurspartikel (Himmelmann 1997; Abraham 1991; Fernandez-Vest 2000)

${ }^{6}$ Das World Lexicon of Grammaticalization (Heine-Kuteva 2002), obwohl nach Diessel entstanden, ist in der Behandlung der Deiktika äußerst knapp.

${ }^{7}$ Die Liste dient der möglichst erschöpfenden Erfassung postulierter Richtungen funktionalen Wandels deiktischer Elemente. Einige der angeführten Funktionsverschiebungen sind umstritten - dies wurde mit einem Fragezeichen gekennzeichnet - , es geht in dieser Arbeit jedoch in erster Linie nicht um die Richtigkeit der einzelnen Daten, sondern darum, dass die angenommenen Grammatikalisierungsketten als zumindest möglich gelten. 
Wie aus der Liste hervorgeht, wurden in der uralistischen Literatur, ohne den Terminus Grammatikalisierung zu verwenden, Zusammenhänge zwischen Demonstrativpronomina und Determination (Akkusativsuffix im Ungarischen, Prädestinativsuffix im Nord-Samojedischen, Translativsuffix im Ostseefinnischen) sowie indexikalischen Ausdrücken ('vorne', 'drüben', fi. Ordnungszahl ensi) bereits postuliert. Die folgende Beschreibung knüpft an einige Ergebnisse aus diesem Bereich an.

1.5. Das Korpus, auf dem folgende Beschreibung beruht, bilden die von Kazis Labanauskas herausgegebene Textsammlung (Labanauskas 2001), sowie die in der Chrestomathia Nganasanica (Wagner-Nagy 2003) publizierten Texte. Die meisten Aufzeichnungen stammen aus den 1970-er Jahren, die neuesten sind in den letzten 10 Jahren verfasst worden, sodass das Korpus diachron gesehen als ein Idiom betrachtet werden kann. Im Falle von Sprachen mit einer langen schriftlichen Tradition lassen sich die einzelnen Stadien von Grammatikalisierungsprozessen verfolgen und die der Reanalyse zugrunde liegenden Kontexte erfassen und datieren - dies ist anhand des vorliegenden Korpus aufgrund des relativ kurzen Zeitraumes, aus dem die Texte stammen, jedoch kaum möglich. So stützt sich die folgende Beschreibung auf Rekonstruktionen aufgrund von synchron ermitteltem Sprachmaterial, indem sie sich der Methoden der historischvergleichenden Sprachwissenschaft bedient. Ergänzt wird die Methodik durch Erkenntnisse pragmatisch-funktionaler Ansätze.

\section{Die deiktischen Stämme im Nganasanischen}

Aus funktionaler Sicht verfügt das Nganasanische über ein dreigliedriges Paradigma. Es unterscheidet zwischen proximaler, medialer und distaler Deixis (Tabelle 1).

Die proximalen Formen werden von dem Stamm am(ə)- gebildet. Janhunen führt diesen Stamm auf PS * äm- $\left(*^{*} m\right)$ zurück, den er als eine Ableitung des Demonstrativstamms ə < PS * $\ddot{a}$ - $\left(\sim *^{*} \sim *_{3}\right)$ erklärt (Janhunen 1977, 19). Das Ableitungsmorphem - $m$ (ə) ist möglicherweise der Reflex des uralischen Pronominalbildungssuffixes *-mз (Majtinskaja $1974, \S 381){ }^{8}$

\footnotetext{
${ }^{8}$ Jedoch erwähnt Majtinskaja unter den Reflexen von ${ }^{*} m_{3}$ lediglich Beispiele aus dem finnisch-ugrischen Zweig, nämlich aus dem Ostseefinnischen und Obugrischen.
} 
Tabelle 1

Paradigmen der nganasanischen Demonstrativformen

\begin{tabular}{|c|c|c|c|c|c|c|}
\hline & proximal & \multirow{2}{*}{\multicolumn{2}{|c|}{$\begin{array}{c}\text { medial/anaphorisch } \\
t \partial-\end{array}$}} & \multicolumn{3}{|c|}{ distal } \\
\hline & әm(ә)- & & & & & $m-$ \\
\hline Abs & әтә & \multicolumn{2}{|l|}{ təว } & takəə & & \\
\hline $\begin{array}{l}\text { Abs } \\
\text { (Präsentativ) }\end{array}$ & әтәпіә әтәи́іә & \multicolumn{2}{|l|}{ - } & taania & & \\
\hline Attr Abs & amti(ra) & \multicolumn{2}{|l|}{ tati(ra) } & - & & \\
\hline Adj & amlad' $i \sim$ ablad' $i$ & \multicolumn{2}{|l|}{ tarad'i } & - & & \\
\hline $\mathrm{Adv}$ & (ә)mi"ia & \multicolumn{2}{|c|}{ təńi"ia/təni } & - & & \\
\hline Lat & $\begin{array}{l}\text { (ә) } m i " i a \sim \\
\text { วтәйi/วməd'a }\end{array}$ & tanda & təńi"ia/təni & & tabə & taba" $a$ \\
\hline Loc & әmni & tənu/tani & təńini & & \multicolumn{2}{|c|}{ tamnu $\sim$ tamni } \\
\hline El & amkata & tagata & təńiðə & & \multicolumn{2}{|c|}{ tamkata } \\
\hline Prol & әтәәпі & tәтәәni & tәи́ітәәпі & & \multicolumn{2}{|c|}{ tатәәпи } \\
\hline
\end{tabular}

Janhunens Rekonstruktion beruht auf den Angaben Castréns (1854/ 1969). In dessen Aufzeichnungen aus den 1840-er Jahren finden wir die Wortfamilie in der Form mit einem prothetischen $\eta$-, bei Castrén *:

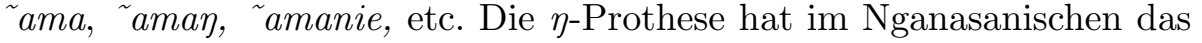
anlautende PS $\ddot{a}$ - aufgelöst. Diese Prothese wurde jedoch bei allen anderen Wörtern, die bei Castrén mit anlautendem $\eta$ - belegt sind, bewahrt mit Ausnahme des Demonstrativstammes am(ə)- (Tabelle 2).

\section{Tabelle 2}

\begin{tabular}{|c|c|c|c|}
\hline PS & Castrén & heute & Bedeutung \\
\hline$* \ddot{a} m\left({ }^{*}{ }_{3} m-\right)$ & $\sim a m a$ & әтә & 'dieser' \\
\hline$\ddot{a} m \ddot{a} j$ & حameai & пamiaj & 'anderer' \\
\hline 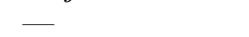 & $\hat{a} m u$ & пәти & 'Tabak' \\
\hline$\ddot{a} m\left(*^{*} 3 m-\right)+{ }^{*}-t V$ & حamti & amti & 'dieser' \\
\hline$a ̈ m t \hat{~}$ & $\sim a m t a$ & qamtə & 'Horn' \\
\hline
\end{tabular}

Im heutigen Nganasanisch kommt ə- im Wortanlaut selten vor. Abgesehen von drei Diskurs-partikeln die onomatopoetischen Ursprungs oder spätere Entlehnungen sein können und daher eine unregelmäßige Entwicklung aufweisen und dem Demonstrativstamm am(ə)- gibt es im Nganasanischen heute nur vier Morpheme mit einem a im Anlaut. Die Entwicklung des Demonstrativstammes bildet auch in dieser Wortgruppe eine Ausnahme: Es gibt keine weiteren Beispiele dafür, dass prothetisches 
$\eta$ - getilgt wird. In zwei Fällen geht anlautendes ə- auf einen Vokal zurück, die anderen beiden sind bei Castrén nicht belegt (Tabelle 3).

\section{Tabelle 3}

\begin{tabular}{|c|c|c|}
\hline Castrén & heute & Bedeutung \\
\hline $\begin{array}{l}\text { iji'a, Augm. von isi 'älterer Bruder' } \\
\text { oder Asa, pl. Aija' 'Tunguse, Dolgane, } \\
\text { eig. jüngerer Bruder' }\end{array}$ & ว"ว & $\begin{array}{l}\text { 1. 'älterer Bruder'; } \\
\text { 2. 'Onkel (jüngerer Bruder } \\
\text { des Vaters)' }\end{array}$ \\
\hline atea & & 'Pflicht, Schuld' \\
\hline- & aliga & $\begin{array}{l}\text { 1. 'Schaden, Nachteil'; } \\
\text { 2. 'schlecht, unanständig', }\end{array}$ \\
\hline 一 & arəkərə & 'schön, wunderbar' \\
\hline
\end{tabular}

Anhand der Belege - und wenn man von der Richtigkeit der Castrénschen Aufzeichnungen ausgeht - muss man annehmen, dass die Wortfamilie des nganasanischen proximalen Demonstrativums am(ə)- eine formelle Anomalie aufweist. Es ist bislang ungeklärt, warum das protetische $\eta$ dieses Morphems wieder getilgt wurde.

Somit bleibt auch unsicher, ob das Vorderglied des nganasanischen əm- tatsächlich bis auf das Uralische zurückgeht, ${ }^{9}$ eine typologische Parallele zu vokalisch anlautenden Demonstrativa in weiteren uralischen Sprachen bildet oder eine Entlehnung ist.

Die beiden $t$-Stämme: den anaphorischen Stamm tz(-) (bei Castrén ta- S. 594) und den distalen tam- (ebenso bei Castrén ebd.) führt Janhunen auf zwei protosamojedische Formen zurück, nämlich auf PS *tวิ(-), bzw. PS *tä(-) *te(-) (Janhunen 1977).

Der tə-Stamm weist zwei Kasusparadigmata auf: Zusätzlich zur pronominalen Bildungsweise, der der absolute Stamm zugrunde liegt, gibt es ein adverbiales Paradigma, das aus dem durch das Lokalkasussuffix erweiterten Stamm təńi- gebildet wird.

Im distalen Paradigma gibt es die beiden Stammmorphemvarianten ta(a)- und tam-. Die Stammvariante tam-kommt nur in den sekundären Casusformen vor, eine adjektivische oder modale Form (Aspektdeixis) weist das Paradigma nicht auf. Es stellt sich die Frage, in welchem Verhältnis diese beiden Morphemvarianten zueinander stehen: Gehören sie historisch zusammen, ist die längere eine Ableitung aus der kürzeren? Die längere Stammvariante ist laut Janhunen eine Ableitung des Demonstra-

${ }^{9}$ Wenn Janhunens Etymologie richtig ist, und Nganasanisch əmə ein Reflex des PS

$* \ddot{a}-\left(? \sim *_{e-} \sim *_{3-}\right)$ ist, dann kann die Rekonstruktion aufgrund der Angaben von Rédei (1988-1991) bis zum Uralischen zurückgefürht werden. 
tivstamms * tâ durch das oben bereits erwähnte Suffix - $m$, die auf das PS, oder möglicherweise auf das Uralische ${ }^{*}{ }_{-} m 3$ zurückgeht.

Castrén belegt die Form des präsentativen Demonstrativs mit kurzem $a$ als tanie ${ }^{10}$ und ordnet es sowohl phonologisch als auch semantisch ('jener, der bekannte, Lat. ille') unter dem Stamm ta- ein (a.a.O. S. 358). ${ }^{11}$ Auffällig ist auch, dass die heute belegte Form taaniz wegen Fehlens der medialen Form in binärer Opposition zu dem proximalen əməniə steht. Dies spricht auch dafür, dass Castréns Schreibweise korrekt ist und die Form taaniz etymologisch mit dem medialen Demonstrativstamm zusammenhängt. Die Dehnung des Vokals bleibt ungeklärt, vermutlich erfolgte sie aufgrund von Analogiewirkung durch das distale Paradigma.

Die Form takəə ist möglicherweise sekundär aus dem Symbolfeldausdruck U taka- 'Hinterraum, das hintere' (Rédei 1988-1991, 506-7), PS *täkâ 'das Hintere' entstanden (Janhunen 1977, 154), erweitert durch das Adjektivbildungssuffix -ə. Die ursprüngliche Bedeutung des distalen Demonstrativums ist 'das hintere, das hinten befindliche'. Die Form ist im heutigen Nganasanisch auch in der ursprünglichen Bedeutung belegt.

Da die Ableitung takəə möglicherweise bereits im Uralischen stattgefunden hat, und die Form taaniz - wie oben gezeigt - auf den medialen Stamm zurückgeht, kann man feststellen, dass im Nganasanischen die distalen Demonstrativa sekundär entstanden sind und dass die heutigen Formen der nganasanischen Demonstrativa etymologisch auf zwei Stämme zurückgeführt werden können.

Synchron betrachtet kann man hingegen drei funktionale Gruppen der nganasanischen Demonstrativa unterscheiden. Während der medialanaphorische Stamm tə- auschließlich im Textraum angesiedelt ist und der Fokuskontinuierung dient, werden die proximalen und distalen Formen exophorisch oder am Phantasma verwendet. Die proximale Form kann auch ana- und katadeiktisch verwendet werden, und ist das sprachliche Mittel der Neufokussierung. ${ }^{12}$

\footnotetext{
${ }^{10}$ Dagegen bezeichnet Castrén die langen Vokale mit $\hat{a}$ (a.a.O. S. 3).

${ }^{11}$ An gleicher Stelle erwähnt Castrén, dass „, amang, tanie im attributiven Verhältnis mit amte, tati vertauscht zu werden pflegen."

${ }^{12} \mathrm{Zu}$ den Begriffen Fokuskontinuierung und Neufokussierung s. Ehlich (1982).
} 


\subsection{Die Grammatikalisierungskette anaphorisches Pronomen $\rightarrow$ Personendeixis $\rightarrow$ Personalsuffix $\rightarrow$ Determinans}

\subsubsection{Anaphorisches Pronomen $\rightarrow$ Px3sg}

Der wesentliche funktionale Unterschied zwischen Personaldeixis (Personalpronomina der 1. und der 2. Person) und Objektdeixis (Personalpronomen der 3. Person) schlägt sich auch in der lexikalischen Form und auf der morphosyntaktischen Ebene nieder. ${ }^{13}$

Die formale Ähnlichkeit zwischen Pronomina der dritten Person und anaphorischen bzw. deiktischen Pronomina ${ }^{14}$ liegt in der funktionalen Affinität der beiden Kategorien begründet. In der uralischen Fachliteratur geht man davon aus, daß das protouralische Personalpronomen der dritten Person, rekonstruiert in der Form * ${ }^{*} 3$, auf ein anaphorisches Pronomen zurückgeht. Die beiden Formen dürften sich jedoch bereits im Protouralischen voneinander unterschieden haben; so bleibt die Annahme, obwohl wahrscheinlich, dennoch hypothetisch (Hajdú-Domokos 1987, 218). Ebenso hypothetisch sind auch die Annahmen über den $\mathrm{Zu}-$ sammenhang zwischen Personaldeixis und Raumdeixis allgemein (ebd.).

Auch das nganasanische Personalpronomen der dritten Person siti wird auf ein anaphorisches Pronomen zurückgeführt. Es wird mit folgenden Reflexen in Zusammenhang gebracht: ens. sed'eo 'der, jener', kam. še 'der dort' / ostjV ti, O śi 'id.'; tscher. sedə 'der, jener'; mord. śe 'jener, dieser da'; fi. se : si- 'es, jener, der' (Majtinskaja 1974, 284; Collinder 1965; Janhunen 1981, 269; Rédei 1988-1991, 34; Hajdú 1990, 3; Itkonen -Kulonen 1992-2000, 163).

Obwohl die Zugehörigkeit einiger Reflexe — so des Tscheremissischen (Collinder 1965; Rédei 1988-1991; Hajdú 1990; Itkonen-Kulonen 1992-

\footnotetext{
${ }^{13}$ So besteht z. B. im ungarischen pronominalen System bei den ersten beiden Personen eine lexikalische Opposition zwischen Singular- und Pulralformen (én:mi, $t e: t i)$, die Pluralform der 3. Person wird jedoch „regelmäßig“, d. h. hier analytisch nach dem nominalen Paradigma gebildet (ő:ők). Im Estnischen zeichnen sich die Pronomina der dritten Person durch das suppletive Paradigma tema: nema aus. Zudem existieren - wenn auch vereinzelt - pronominale Systeme, in denen die lexikalische Form des Pronomens der ersten Person zu denen der zweiten und dritten Person in Opposition zu setzen ist. Letztere sind jedoch in der Regel sekundäre Formen, die auf einen Nominalstamm mit lexikalischer Bedeutung zurückgehen, wie z. B. im Nenzischen, wo die Pronomina der zweiten und dritten Person auf das Nomen nы до 'Körper' zurückgehen (Sebestyén 1964).

${ }^{14}$ Zum Funktionswandel 'dies $>$ er' vgl. estn. tema, tscher. tudo, fi. se, fr. $i l<$ lat. *ille 'der, dieser', got. is, ahd. er - lat. is 'der, dieser' (Hajdú-Domokos 1987, 218).
} 
2000) und des Kamassischen (Collinder 1965; Rédei 1988-1991; Itkonen -Kulonen 1992-2000) - häufig angezweifelt wurde, hielt man an der bis zur uralischen Grundsprache zurückgehenden Etymologie fest. ${ }^{15}$ Lediglich über die Qualität des anlautenden Konsonanten wurde debattiert: Setälä (1902, 268-9) und ihm folgend Janhunen $(1981,51)$ rekonstruieren einen palatalen Spiranten $\left({ }^{*} s ́\right)$, wogegen Paasonen $(1906,211)$ und Rédei (1988-1991) von einer Affrikate $\left({ }^{*} c\right)$ ausgehen.

Für das Protosamojedische wird die Form * se rekonstruiert. Da jedoch das kamassische anlautende $\check{s}$ - regelmäßig auf PS ${ }^{*} k$ - zurückgeht (Sammallahti 1988, 498), gehört kam. še 'der dort' nicht in diese Zusammenstellung. Enzisch sedeo ist wegen seiner Bedeutung 'der vorherige' kein sicheres Glied der Etymologie. Zieht man die Konsequenzen aus den lautlichen und semantischen Unregelmäßigkeiten, und schließt man die nicht zugehörigen Formen von der Zusammenstellung aus, bleibt auf der samojedischen Seite allein nganasanisch siti. Wie aus folgender Tabelle hervorgeht, kann man die Urform des nordsamojedischen Personalpronomens der dritten Person wegen fehlender Entsprechungen im Nenzischen und Enzischen nicht rekonstruieren.

Tabelle 4

Personalpronomina der 3. Person in den samojedischen Sprachen

\begin{tabular}{|c|c|c|c|c|}
\hline & sg. & du. & pl. \\
\hline \multirow[t]{3}{*}{ NSam } & Nganasanisch & (C) sete / siti & (C) seti / siti & (C) seten / sitin \\
\hline & Nenzisch & [sekundär] & {$[\ldots]$} & {$[\ldots]$} \\
\hline & Enzisch & {$[\ldots]$} & {$[\ldots]$} & {$[\ldots]$} \\
\hline \multirow[t]{3}{*}{ SSam } & Selkupisch & tepp & tep $\bar{a} q i$ & tepit \\
\hline & Kamassisch & teo, $d i$ & dišide & dizen \\
\hline & Matorisch & $t i(? \operatorname{tin})$ & & $t i(? \operatorname{tin})$ \\
\hline
\end{tabular}

Quellen: Vértes (1967), Wagner-Nagy (2003), Helimski (1997, 147)

Wenn man der Rekonstruktion der gemeinsamojedischen Form jedoch die südsamojedischen Formen zugrunde legt, ergibt sich die Form ${ }^{*} t V$-, von der aber nganasanisch siti nicht abgeleitet werden kann, denn die Fortsetzung von PS $t$ - im Nganasanischen ist $t$ - oder $t$ - $[\check{c}]$.

${ }^{15}$ Allein bei Janhunen wird die Zurückführung bis zum Uralischen in Frage gestellt, jedoch nicht für das Samojedische: Nganasanisch siti gilt als sichere Fortsetzung des Demonstrativpronomens, nicht jedoch finnisch-permisch ?* śs (Janhunen 1981, 51). 
Es finden sich auch innersprachlich keine Formen, die mit siti etymologisch zusammenhängt. Daher stellt sich die Frage, ob ein anaphorisches Demonstrativpronomen in einer Sprache keine weitere Spur hinterlassen haben kann. Man denke an das finnische anaphorische Pronomen se, das im kolloquialen Gebrauch in der Rolle des Personalpronomens der dritten Person geläufig ist - also durchaus vergleichbar mit dem vermuteten Entwicklungsszenario von siti - und ein vollständiges Kasusparadigma (se, sen, sitä, siihen etc.) sowie andere Derivate (siksi, sek̈̈... että...) aufweist. Aus lautlichen Gründen und mangels innersprachlicher und samojedischer Entsprechungen scheint somit die Herleitung von siti aus dem anaphorischen Pronomen unwahrscheinlich.

Eine Alternative bietet Helimskis Etymologie. ${ }^{16}$ Demnach gehört nganasanisch siti aufgrund regelmäßiger Lautentsprechungen mit folgenden Pronominalstämmen zusammen: dem Genitiv- und Akkusativstamm der Personalpronomina im Nenzischen und Enzischen sowie dem Akkusativstamm der Personalpronomina der 1. und 2. Person im Selkupischen (Janhunen 1977, 70-1, Helimski 1982, 90-3). Die genannten Reflexe gehen auf NSam. ${ }^{*} k i t^{1}$ (PSam. ${ }^{*} k i$ ) bzw. U *ke 'Ähnlichkeit, Bild' zurück.

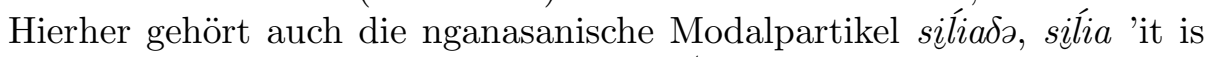
not clear whether, I don't know' < '*kit ${ }^{1}$-only-for' (Helimski 2003) (etwa 'nur zur Ähnlichkeit'). Möglicherweise geht auch das nganasanische Interrogativpronomen sili 'wer' auf denselben Stamm zurück, unklar ist hier jedoch die Endung $-l i$ ? $<-r i$.

Im Nganasanischen wurde das Wort in gleicher Lautform si " : siti zudem auch in der ursprünglichen Bedeutung bewahrt (Kosterkina et al. 2001, 159). Die genannten Reflexe gehören zur Gruppe derjenigen Pronomina und Abstrakta, die sich sich durch semantische Entleerung aus Nomina mit konkreter Bedeutung entwickelt haben.

\subsubsection{Personalpronomen $\rightarrow$ Personalsuffix?}

Wenn auch die Grammatikalisierungskette anaphorisches Pronomen $\rightarrow$ Personalpronomen der 3. Person sg. für das Nganasanische nicht durch die Etymologie der heutigen pronominalen Form bewiesen werden kann, so scheint es doch Hinweise auf eine entsprechende Entwicklung zu geben.

\footnotetext{
${ }^{16}$ Vorgestellt im Rahmen des 4. Uralischen Workshops von Budapest (BUM IV). Die folgende Darstellung beruht auf der dort präsentierten Tischvorlage.
} 
Tabelle 5

Nganasanische Personalsuffixe, die ein Segment $-t V$ - enthalten

$\begin{array}{lccc} & \text { sg.N/du.,pl.NGA; obj./sg. } & \text { sg.A } & \text { adv./sonst. } \\ \text { 3sg.: } & -\mathrm{TU} & -\mathrm{MTU} & -\mathrm{NTU} \\ \text { 3du.: } & -\mathrm{TI}^{\mathrm{c}} & -\mathrm{MTI}^{\mathrm{c}} & -\mathrm{NTI}^{\mathrm{c}} \\ \text { 3pl.: } & -\mathrm{TUn} & -\mathrm{MTUn} & -\mathrm{NTUn}\end{array}$

In der überwiegenden Zahl der Personalsuffixparadigmen kann man für die dritte Person ein Segment - $t U$ - (-tu/-ti) unterscheiden, das dem Lautbild des Demonstrativpronomens sehr nahe steht, wenn auch nicht identisch mit diesem ist. Das Personalsuffix geht auf protosamojedisch *-tA (< uralisch *-sA) zurück (Mikola 1988, 240; Helimski 1997, 142), und unterscheidet sich daher nicht nur im Konsonantismus, sondern auch im Vokalismus von den Demonstrativstämmen: sowohl vom anaphorischen Pronomen PS *tวิ(-), als auch vom distalen Demonstrativpronomen PS ${ }^{*} t \ddot{a}(-) \sim * t e(-)$. Dass das uralische Personalpronomen der 3. Person sg. ${ }^{*} s 3$ auf einen Demonstrativstamm zurückhgeht bleibt also eine Vermutung, während die Verbindung zwischen dem Personalsuffix *-sA und dem Personalpronomen * sз sehr wahrscheinlich zu sein scheint. Im Samojedischen oder in einer früheren Sprachstufe wurde das ehemalige Personalpronomen der 3. Person $U^{*}$ ss zum Personalsuffix agglutiniert. In einer späteren Sprachstufe wurde das Personalpronomen durch eine periphrastische Form mit der Bedeutung 'sein Bild, sein Antlitz' ersetzt (S. 2.1.1).

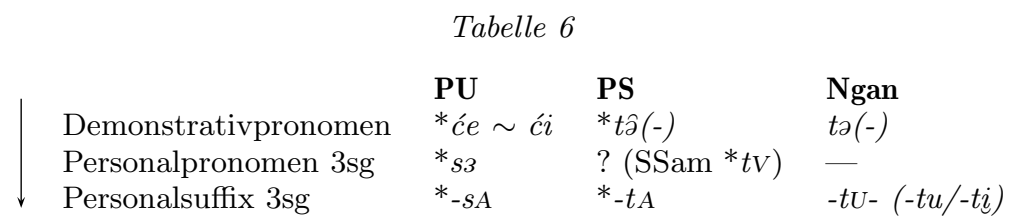

\subsubsection{Personalsuffix (Px2sg, Px3sg) $>$ determinierendes Suffix}

Das Nganasanische hat keinen bestimmten Artikel. In determinativer Funktion werden die Possessivsuffixe der zweiten und dritten Person verwendet. Da ein Possessivsuffix stets ein bestimmtes Relationsverhältnis ausdrückt, identifiziert es eindeutig die Referenz seines Bezugswortes. Diese Leistung macht es möglich, dass dieses Suffix auch auf Kontexte übertragen werden kann, in denen es allgemein um Determination geht.

Die Allomorphe der proximalen und medial-anaphorischen Pronomina des Nganasanischen əmə, əmti, əmtirə bzw. təə, təti, tətirə sind 
zweimal in der Folge durch Possessivsuffixe erweitert worden. In der Endung der Form tati identifiziert man das Possessivsuffix der 3. Person - $t i$, zu dem nach der semantischen Entleerung des Suffixes ein weiteres determinierendes Element, nämlich das Possessivsuffix der zweiten Person -rə hinzugefügt wurde (Wagner-Nagy 2003, 97).

Dagegen schlägt Helimski (mündliche Mitteilung) eine andere Erklärung vor, die der abweichenden genitivischen Form təndə Rechnung trägt. Er hält eine Formbildung durch Reduplikation für möglich, wobei aus der ursprünglich reduplizierten genitivischen Form tən-tən die heutige Form təndə nach der Auslautreduktion und der Stimmhaftwerdung von $t$ - nach dem nasalen - $n$ regelmäßig entstanden ist. Das Reduplikationsprinzip läßt sich jedoch nicht auf die Ableitungen von əmə- übertragen. Führt man beide Erklärungen zusammen, kann man annehmen, dass zunächst der anaphorisch gebrauchte Stamm durch Reduplikation erweitert wurde. Die reduzierte Form wurde als Possessivsuffix reinterpretiert (da Possessivsuffixe in determinativem Gebrauch durchaus geläufig sind, erscheint die Reinterpretation möglich) und mit der Zeit auf die proximalen Formen übertragen.

\subsubsection{Demonstrativpronomen $>$ Determinans abgeschwächt demonstra- tiven Charakters}

In nganasanischen Texten neueren Datums, die der Oralität nahe stehen, fällt der häufige Gebrauch des Demonstrativums amə/amti und des anaphorischen tati in attributiver Stellung auf. Ihr Erscheinen selbst vor Eigennamen scheint durch die determinierende Funktion nicht begründet.

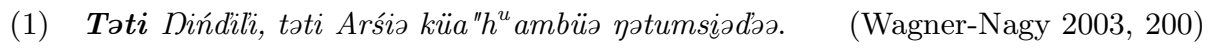
'DEM (der) Dińdili, er kam auf die Welt als Arsia am Sterben lag.'

Eine derart markierte Fokuskontinuierung erscheint im Vergleich zu weiteren, früher aufgezeichneten und zu primär schriftlichen Texten redundant. Noch auffälliger ist die Verwendung von amə, wenn man davon ausgeht, dass es zum Zweck der Neufokussierung eingesetzt wird. Eine mögliche Erklärung dieser Verwendungsweise, die jedoch am gesprochenen Sprachmaterial überprüft werden sollte, ist, dass die deiktische bzw. anaphorische (fokuskontinuierende) Funktion beider Pronomina abgeschwächt ist. Dies würde eine Übersetzung ins Deutsche mit dem bestimmten Artikel rechtfertigen. Es bleibt zu klären, ob es sich hier um den Ansatz eines Grammatikalisierungsprozesses handelt, der zur Her- 
ausbildung eines Artikels führen könnte, oder ob diese Wörter weitere, diskursgliedernde Funktionen erfüllen. Der Frage wird in Punkt 2.5 nachgegangen.

\subsubsection{Personalpronomen 3sg $>$ Determinativsuffix $>$ Prädestinativsuffix?}

Der Prädestinativ ist eine den nordsamojedischen Sprachen gemeinsame nominale Kategorie, mit dativischer und essivischer Semantik, die bezeichnet, das ein Objekt für jemanden gedacht, geschaffen oder gehalten wird. Im Nganasanischen hat das Suffix die Form - Tz. Das Deklinationsparadigma beschränkt sich auf den Nominativ, Akkusativ und Genitiv. Morphosyntaktisch folgt das Prädestinativsuffix den Derivationssuffixen und geht den Kasussuffixen voran. Der deiktische Ursprung des Suffixes scheint aus semantischer Sicht wahrscheinlich.

Einige Forscher (z.B. Ago Künnap, Tibor Mikola) führen das Prädestinativsuffix, das für das Nordsamojedische in dieser Form rekonstruiert wird, auf das Personalsuffix der 3sg zurück. Diese Annahme ist jedoch, wie Janhunen (1998) gezeigt hat, aus phonologischen und morphosyntaktischen Gründen nicht haltbar.

\subsection{Lexikalisierung von $t a$ - in adverbialen Konstruktionen}

\subsubsection{Lokalisierung $\rightarrow$ habeo-Konstruktion}

Durch Kontraktion des anaphorischen Stammes und des Existenzverbs ist die Form taiśa entstanden, die formell die gleiche Struktur aufweist wie deutsch dasein. Die Bedeutungen von taiśa können auf einer Skala von konkreten (semantisch durchsichtigen) Verwendungen bis hin zum Abstrakten eingeordnet werden: 'vorhanden sein, zu finden sein, leben, haben, besitzen / иметься, быть (у кого-л.)'. Während der Demonstrativstamm tə ursprünglich eine Verbalergänzung mit der Funktion der Lokalisierung war, wurde in Kontexten, in denen Possessivrelationen im Verbargument auftauchten, die gesamte Struktur als habeo-Konstruktion reinterpretiert:

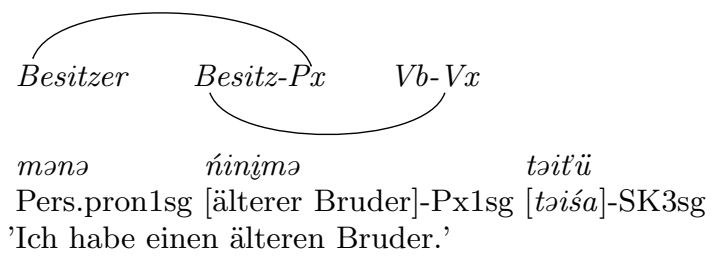

(Wagner-Nagy 2003) 
Die syntaktische Tiefenstruktur (a) wurde reinterpretiert als syntaktische Tiefenstruktur (b):

(3) (a)

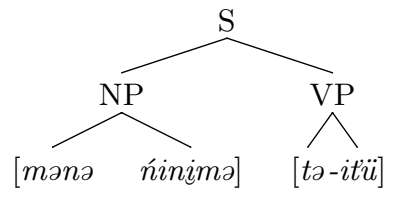

(b)

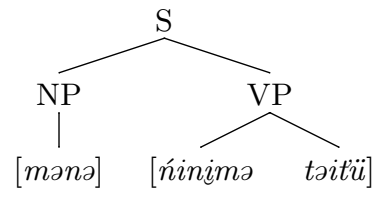

Auch die lokativische Form des anaphorischen Pronomens geht eine solche Verbindung mit dem Existenzverb ein: tənijśa '(bei jdm.) vorhanden sein' $<$ təni+ iśa 'da' + 'sein' :

(4) bənsagi nigi yojbu ńińi arəkərə, kartako" namtə" tənijtu". alle-du Frau-du Kopf Postpos-Loc schön verzweigt Horn-pl [tənijśa]-SK3pl 'Auf den Köpfen beider Frauen waren schöne, verzweigte Hörner.'

Beide Verben können lokativische Argumente aufnehmen, die sogar durch ein weiteres Demonstrativum besetzt sein können:

(5) amni tainu" lekira"?

hier [təiśa]-SKInterr3pl Arzt-pl

'Gibt es hier Ärzte (einen Arzt)?'

(Wagner-Nagy 2003, 195)

tə- und təni füllen die vom Verb eröffnete Leerstelle für eine Lokalergänzung nicht aus. Dies ist ein eindeutiges Zeichen der semantischen Entleerung.

\subsubsection{Idiomatisierung von tərəd'i, təńii"ia 'so' in adverbialer Konstruktion: ein erster Schritt zur Lexikalisierung?}

Dem Lexem təńi"iaiśa liegt die Adverbialkonstruktion təńi"ia + iśa zugrunde: Die Adverbialbestimmung təńi" $i$ is ist die lativische Form des anaphorischen Pronomens, und iśa ist der Infinitiv des Existenzverbs.

(6) tərədii iśa, təñi"iaiśa (təńii"iaiśa) 'so seiend' > 'daher'

Das Nganasanische weist nur wenige Satzkonnektoren auf, konditionale und kausale Relationen zwischen koordinierten und subordinierten Gliedsätzen bleiben unmarkiert.

Die satzwertige Konstruktion mit der Bedeutung 'so seiend' kann in verschiedenen Kontexten erscheinen: 


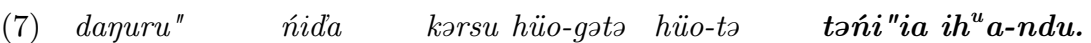
Tundra-Genpl postpLat. Ding Jahr-Elat Jahr-Px3sg so sein-SK3sg 'So ist die Lage auf der Tundra Jahr für Jahr.'

(8) tabńi"ia ihü"ta, sierle ńintu ańi"e".

so sein-Ger-Px2sg Sünde-Px2sg nicht groß

'Wenn du dich so verhältst, begehst du keine großen Sünden.'

(9) təńi"ia ihü", domtukumu"!

so $\quad$ sein-Ger kämpfen-ImpSKVx1pl

'Wenn es so ist, sollen wir kämpfen!'

In (7) wird təńi"ia zwar textdeiktisch verwendet, es besteht jedoch keine logische Verbindung zwischen den Gliedsätzen, während in (8) eine konditionale Relation zwischen den beiden Gliedsätzen herrscht.

Die Konstruktion eignet sich, da sie die anaphorische Komponente təńi"ia enthält, zur metonymischen Übertragung logischer Beziehungen, die zwischen den Gliedsätzen bestehen.

Die Zusammenschreibung spricht dafür, daß die Konstruktion von Sprechern des Nganasanischen als eine lexikalische Einheit empfunden wird. ${ }^{17}$ Die Konstruktion hat jedoch - obwohl sie in der Funktion eines Satzkonnektivs mit kausaler Bedeutung ('so seiend' > 'deshalb') idiomatisiert ist - ihre morphosyntaktische Durchsichtigkeit noch nicht verloren: Das Verb wird konjugiert (s. z.B. (7)), daher kann hier erst das Anfangsstadium der Lexikalisierung festgestellt werden.

\subsection{Funktionswandel im Bereich der Ableitungen von tə-}

Konjunktionen und complementizer weisen eine komplexe Semantik auf. Sie bezeichnen eine logische Relation zwischen zwei Gliedsätzen und beziehen diese Relation anaphorisch auf ein vorab oder kataphorisch auf ein nachfolgend definiertes sprachliches Bezugselement (eine Phrase oder eine Proposition). Die Referenzbestimmung wird in der Regel durch eine deiktische Komponente geleistet.

Es gibt nur eine geringe Zahl von syntaktisch wenig gebundenen nganasanischen Konjunktionen. Der Grund dafür liegt darin, dass diese Kategorie im Nganasanischen eine relativ späte Erscheinung ist.

\footnotetext{
${ }^{17}$ Geläufig ist in gleicher Bedeutung auch die Konstruktion aus Aspektdeixis tərədi $i$ 'so' und Existenzverb iśa. Dass diese Konstruktion nicht kontaminiert wurde, hat möglicherweise prosodische Gründe.
} 


\subsubsection{Die Lexikalisierung von tagata(ta) 'von dort, von da' $>$ 'von da an, danach'}

təgətə ist die elativische Form des anaphorischen Pronomens. Durch metonymische Erweiterung erlangte das Lexem die temporale Bedeutung der Nachzeitigkeit 'danach'. Als der Bedeutungswandel schon eingetreten war, wurde das Lexem durch das Possessivsuffix der dritten Person tə zu tagatati erweitert.

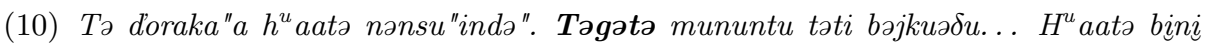

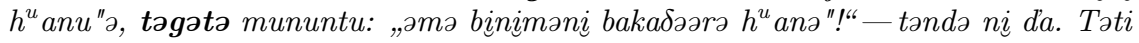
nikü tagatati mununtu: „Tə-tə.“ (Wagner-Nagy 2003)

'Sie hielten bei einem gewundenen Baum an. Dann sagt ihr Mann... Er hat ein Seil am Baum befestigt, dann sagt er: „Stecke deinen Hals in dieses Seil“, — sagt er der Frau. Die Frau sagt danach: „Gut".'

\subsection{2. təbtə 'auch' - ein Fall der Lexikalisierung?}

təbtə ist die soziative Partikel mit der Bedeutung 'auch'. Sie steht überwiegend $^{18}$ vor dem Prädikat. In ihrem Skopus kann sowohl das Prädikat stehen (a) als auch eine topikalisierte Phrase (b), wobei zwischen diesen beiden Fällen strukturelle Homonymie herrscht. Möglicherweise wird der jeweilige Skopus durch suprasegmentale Mittel markiert.

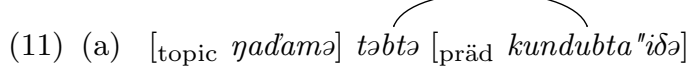
'Mein jüngerer Bruder schlief auch ein.'

(b) [topic mənə] təbtว [präd babij horanantuduom] 'Ich wollte auch wilde Rentiere jagen.'

Die Herkunft von tabtə gilt als unbekannt. Geht man von einer Ableitung aus, bietet sich die Segmentierung tə $+-b t z$ an, da die so ermittelten Segmente bekannte Morpheme des Nganasanischen sind. Im Vorderglied der Ableitung ta kann man das anaphorische Pronomen mit der Bedeutung 'dies, jenes (schon Erwähnte)' erkennen.

Es gibt eine Reihe von Wörtern im Nganasanischen, in denen ein denominales Suffix der Form - $\boldsymbol{b} t \boldsymbol{t}(\boldsymbol{\partial}) /-\boldsymbol{b} \boldsymbol{t} \boldsymbol{a}$ abgegrenzt werden kann: bərəbta 'Äußeres'; nad'abta nadabta 'jüngerer/jüngster [Bruder, Schwester]'; nerəbta 'erster'. Möglicherweise gehört mibtiə 'Sachen/Dinge' in

${ }^{18}$ Vereinzelt kann tabtə auch dem Prädikat folgen. Eine detailliertere Beschreibung seiner Syntax steht noch aus. 
diese Reihe (Katzschmann 2003, A.4.9). Auch das zwiete Glied des heute nicht mehr produktiven Soziativsuffixes - $\boldsymbol{S} \boldsymbol{\partial} \boldsymbol{B T}$, geht auf dieses Suffix zurück. ${ }^{19}$ Vergleicht man die Bedeutung der Ableitungen mit der der Stämme:

(12) Yad'a 'jüngerer'

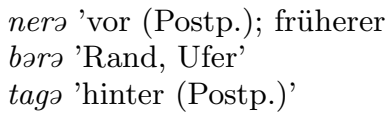

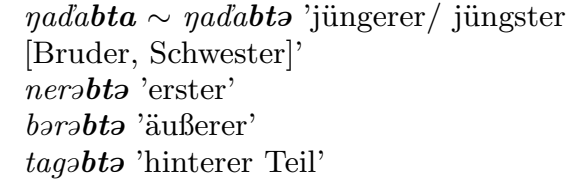

Es fällt auf, dass die letzteren Belege auf ein Adjektivbildungssuffix hindeuten; genauer handelt es sich, wie Katschmann vermutet, um ein Suffix mit limitativer Bedeutung (Katzschmann 2003, A.4.10, ung. kijelölő jelzöi szuffixum - Wagner-Nagy 2003, 127): 'derjenige, der die Eigenschaft X hat, zu X gehörig (wobei X für die Bedeutung des Stammes steht)'. Folglich kann - wenn die vorgeschlagene Segmentierung richtig ist - als ursprüngliche Bedeutung von təbtə 'zu dem/dahin gehörig' postuliert werden, eine semantische Parallele zum deutschen Konnektoren zudem.

\subsection{Modale Bedeutungen}

\subsection{1. - ta(") Modalpartikel des Evidentialis und des Mirativs}

Die Partikel -tə(") gilt in der Fachliteratur als emphatische oder Verstärkungspartikel mit der Bedeutung 'ведь/hiszen/doch'. ${ }^{20}$ In unterschiedlichen Kontexten variiert die Funktion der Partikel zwischen Mirativität (so in (a) und eventuell in (c)) und Evidentialität (b), (d): ${ }^{21}$

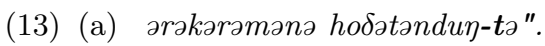

'Du schreibst ja schön!'

(b) matว-tə" tu"omu".

'Wir sind doch/aber ins Zelt gegangen.'

${ }^{19}$ Dieses Soziativsuffix ist zusammengesetzt. Das vordere Segment $-S$ ə ist mit dem ersten Glied des Bildungs-suffixes der nomina loci $-S ə M U$ zu identifizieren. Das zweite Glied dieses Suffixes geht seinerseits auf $-M U<\mathrm{PU} * m a$ zurück (Lehtisalo 1936, 109). Die Semantik des Segments -Sə ist unklar, es scheint jedoch wahrscheinlich, daß die Semantik des Suffixes - BTz ausschlaggebend für die Semantik des Soziativsuffixes 'samt dem Bezeichneten, zusammen mit dem Bezeichneten' ist.

${ }^{20}$ Nach Usenkova $(2003,104)$ „bejahende Partikel“ (утвердительная частиu a), Wagner-Nagy (2003, 173) „nyomósító partikula“ (hiszen), Katzschmann (2003, 160): „Verstärkungspartikel Exklamation" - na dann, los, in Ordnung.

${ }^{21}$ Die Beispiele (a) bis (d) stammen aus Usenkova (ebd.). 
(c) soruli"ə-tə". 'Es regnet ja!'

(d) nərənini" bigaj-tə", nintu bika"kü.

'Vor uns liegt doch/aber ein Fluß, kein Bächlein!'

Die Partikel wird an ihr Skopus klitisiert. Beispiel (13d) deutet darauf hin, dass die Partikel im fokussierten Satz dem Fokus angefügt wird. Im nicht-fokussierten Satz erscheint sie in Endstellung.

Die Zusammengehörigkeit der Partikel - tə(") und des anaphorischen Pronomens kann außer durch das ähnliche Lautbild auch durch funktionale Zusammenhänge gestützt werden. ${ }^{22}$ Das anaphorische -tə greift auf der propositionalen Ebene den aus dem Diskurs bekannten Aktanten auf, wobei die Evidentialitätspartikel hier die Proposition als vom (Sprecher und) Hörer bekannt markiert. Je nach pragmatischem Kontext kann die evidentielle Bedeutung ins Mirativische wechseln:

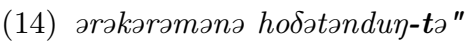

[evid]: 'Du schreibst schön' $\rightarrow$ [mir]: 'Du schreibst schön'

\subsection{2. ta(ə), taวta-Pragma-Idiom}

tə(ə) 'na dann, in Ordnung, einverstanden' ist laut Katzschmann ein Exklamativ, d. h. eine Verstärkungspartikel. Diese Partikel bedeutet Einwilligung bzw. Einverständnis, wie die folgenden Beispiele aus Katzschmann illustrieren (um den Kontext zu verdeutlichen, werden längere Passagen übersetzt):

(15) Nach einiger Zeit sagte der jüngere zu seinem älteren Bruder: Ich will auf die Jagd gehen. Ich nehme einige Rentiere mit und unsere Schwester als Treiber unserer Rentiere. Der ältere Bruder sagte: $\langle 2785 \mathrm{ff}\rangle$

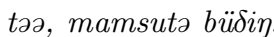

'In Ordnung, geh, wenn du willst.'

(16) Nach einigen Monaten sagte ihr Ehemann: Hast du die Worte meiner Mutter (auch) nicht vergessen? Wir werden zu ihr zu Besuch gehen. Sprich zu deinem älteren Bruder. Der ältere Bruder sagte: $\langle 2960 \mathrm{ff}\rangle$

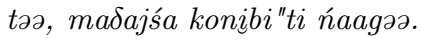

'In Ordnung, (es wäre) gut wenn ihr zu Besuch geht.'

${ }^{22}$ Die Erscheinung ist auch typologisch belegt. Vgl. Willett (1988). 
(17) Das ältere [Mädchen] sagte: Hier werde ich dich zurücklassen! Dann werde ich gehen (und) mein gutes Leben suchen. Die jüngere [Schwester] sagte: tәа, әтә kouki"әm.

'In Ordnung, ich werde hier bleiben.'

Die Form taətə scheint eine Formvariante zu sein. Entweder handelt es sich hier um eine reduplizierte oder um eine emphatische Form, erweitert durch den Px2sg.

In der überwiegenden Zahl der Belege erscheint die Partikel satzinitial, aus der syntaktischen Struktur ausgeklammert. Allein in der Konstruktion ńaagəə tәə (ńaagəə 'gut') wird təə nachgestellt:

(18) Die Alte hörte auf zu schweigen (und) rief: Schneidest du wieder die Worte deines Kindes ab? Wenn er gehen will, soll er gehen! Der Alte sagte:

ńaagəə tәə, kərbubü"te, koni"!

'Na gut, wenn du willst, gehe!'

Es ist leicht nachzuvollziehen, dass die Proposition ńaagəə təə 'das ist gut' (oder 'es ist gut') (ńaagəə 'gut') sich als Ausdruck des Einverständnisses eignet. Problematisch bei dieser Deutung ist jedoch die untypische Nachstellung des Topiks.

Wenn man ńaagəə təə als korrekten nganasanischen Satz mit propositionaler Bedeutung akzeptiert, dann dient dieser Satz möglicherweise als Schlüsselkontext, der idiomatisiert werden konnte. Die zugrunde liegende syntaktische Struktur wurde undurchsichtig, die pragmatische Funktion übernimmt nunmehr der Gesamtausdruck. So konnte auch ein Segment der ursprünglichen Struktur die pragmatische Funktion des Ganzen übernehmen.

Wie kann man dieses Entwicklungsszenario einordnen? Die ursprüngliche deiktische Funktion von təə wurde vollständig aufgehoben. Die neue, pragmatische Bedeutung, die nunmehr auch zur lexikalischen Bedeutung von təə gehört und im Lexikon repräsentiert werden sollte, wurde auf das Zeichen übertragen. Im Falle von ńaаgəə təə erfolgte eine Idiomatisierung, also wurde das Lexikon durch ein Pragma-Idiom erweitert. Je nach (Enge der) Auslegung des Lexikalisierungsbegriffes kann man die Erscheinung als Lexikalisierung annehmen oder ausschließen. Die Beurteilung der Variante təə 'in Ordnung' ist schwieriger: Der Wandel hat hier 
nicht zur Erweiterung des Lexikons beigetragen, sondern zur Erweiterung der lexikalischen (pragmatischen) Bedeutung eines Lexems. ${ }^{23}$

Im folgenden Kontext signalisiert der Sprecher durch die Partikel təə, dass er eine Behauptung seines Gegenübers akzeptiert. Die darauf folgende Proposition schränkt jedoch diese Behauptung ein. So entsteht zwischen den beiden Propositionen eine konzessive Relation, die durch Reanalyse der Partikel zugeschrieben werden kann: ${ }^{24}$

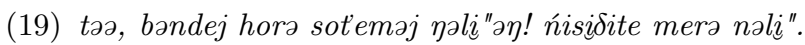

'Na gut, bislang hast du jeden Ewenken besiegt. (Doch) du willst sie nicht (alle) besiegen.'

$\langle 2523-4\rangle$

\subsection{Sprachliches Zeigen $>$ Determinieren $>$ Text- bzw. Diskursgliederung}

\subsubsection{Die Rolle von amti(ra), tati(ra) in der Informationsgliederung des Satzes}

Im Nganasanischen können Phrasen durch Ausklammerung aus dem Satz (Bewegung nach links) topikalisiert werden. Ein anaphorisches Pronomen ersetzt die topikalisierte Phrase im Satz:

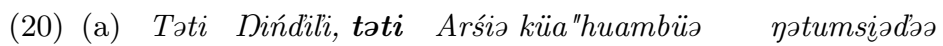
DEM Dińdili DEM Arśiə im.Sterben.liegen geboren-werden-IndPerfVx3sg 'Der Dińdili, der kam auf die Welt, als Arśiə im Sterben lag.'

(Wagner-Nagy 2003, 200)

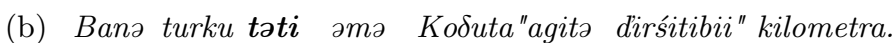
Hund See DEM DEM Dudinka-Elat $120 \quad \mathrm{~km}$ 'Der Hund-See, der liegt 120 km entfernt von (diesem) Dudinka.'

(Wagner-Nagy 2003, 210)

${ }^{23}$ Für die Übertragung einer pragmatischen Bedeutung auf deiktische Morpheme soll hier ein weiteres Beispiel aus dem Ungarischen stehen. Das veraltete ungarische Wort tat, tott, das auf den durch ein Lokativsuffix erweiterten distalen pronominalen Stamm ta- zurückgeht, hat hier die Funktion der verstärkten Behauptung: Monda Xanthus. Vezteg hijtwanfag, nem tudode hogij ezt een wgijan zeretem mijnt ennen magamat. Ees monda Esopus zeretede ez azzonkat? monda Xantus, zeretem tat. 'Xantus sagte: Du [...], weißt du denn nicht, dass ich dies genau so liebe wie mich selbst? Und Esop fragte: Liebst du dieses Weibchen? Xantus sagte: Ich liebe sie wohl'. (Pesti: Fab. 79a)

${ }^{24}$ Diese Entwicklung hat eine weitere Parallele in ungarisch tat: Mykoron az roka giakorta wetne zemere az newften orozlannak hogy ew chak mijndenkoron egyet zywlne, mōda eczer nekij az orozlan Egyet zÿlek tat de orozlant 'Da der Fuchs der Löwin häufig vorwarf, dass sie immer nur ein Junges gebärt, sagte die Löwin einmal zu ihm: Ich gebäre eins, doch das ist ein Löwe.' (Pesti: Fab. 79a) 
Die deiktische Funktion des Pronomens wird hier in eine syntaktische und informationsgliedernde umgewandelt.

In einem weiteren Schritt der Entleerung der deiktischen Funktion werden auch morphosyntaktische Merkmale der Koreferenz aufgehoben: In den weiteren Beispielen kongruiert tati nicht mehr mit seiner Bezugsgröße. Es besteht kein Rückverweis auf die tati vorangehende Phrase, vielmehr gilt təti als Grenze zwischen Topik und Prädikation.

Die topicmarkierende Funktion von təti ist nicht grammatikalisiert, und somit auf der pragmatischen Ebene anzusiedeln: Die lexikalische Markierung des Topiks ist optional und vorwiegend in der gesprochenen Sprache - dort jedoch in einer beachtenswerten Häufigkeit - anzutreffen.

\subsubsection{Endophorische Deixis $\rightarrow$ Textdeixis $\rightarrow$ Diskurspartikel: tati, tətirə}

Betrachten wir die Verwendung von tətirə und tatirə $\operatorname{tahar}^{i} a$ im folgenden Textabschnitt:

(21) 1. Mənə ńimə ńaadətəmənu D'ini"m ${ }^{i} a k u$. 2. Maad’a mənə tərədi ńimə? 3. Taharia

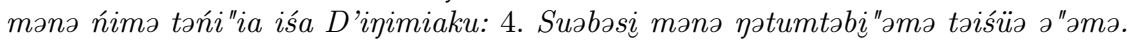

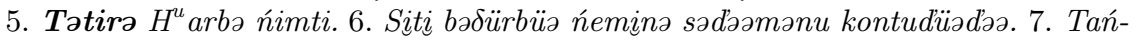

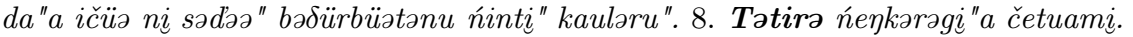

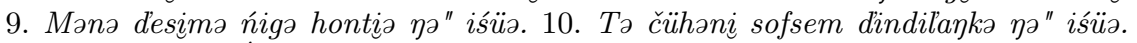

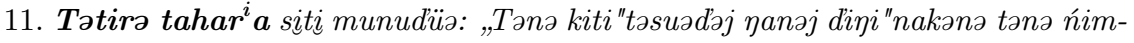

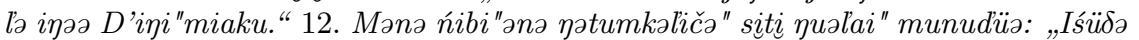

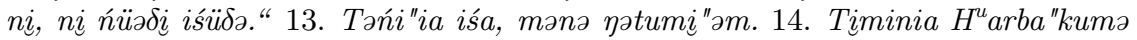

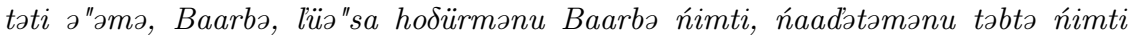

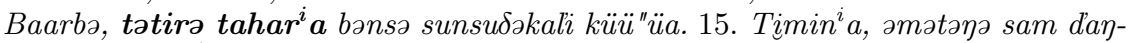

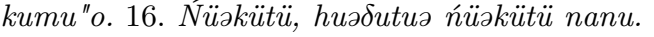

(Wagner-Nagy 2003, 198)

'1. Auf Nganasanisch heiße ich D'ini"miaku. 2. Wieso habe ich solch einen Namen? 3. [Taharia] es kam so: 4. Denn als ich geboren wurde, lebte (noch) mein Onkel. 5. Dieser hieß $\mathrm{H}^{\mathrm{u}}$ arbə. 6. Er kreuzte den Weg von meiner Mutter mit einem Toten. 7. Es ist verboten, vor Schwangeren mit einem Toten den Weg zu überqueren. 8. Dies ist ein sehr starkes Verbot. 9. Mein Vater war ein mächtiger Schamane. 10. Damals war er ein sehr berühmter Schamane. 11. Tətirə taharía sagte er: „Um die bösen Geister zu binden, werde ich dich D'ini"miaku ('kleiner Knoten') nennen." 12. Ich war noch nicht einmal geboren, da sagte er: „Es wird ein Mädchen, eine Tochter wird es." 13. So war es, (so seiend), ich kam zur Welt. 14. Nun unser $\mathrm{H}^{\mathrm{u}}$ arbə, das ist mein Onkel, Baarba, nach russischer Weise ist Baarba sein Name, auf Nganasanisch heißt er ebenfalls Baarba, Tətirə taharía (er) verstarb ohne Nachwuchs. 15. Jetzt, diesen Sommer ist er verstorben. 16. Mit seinem Sohn, mit seinem letzten Sohn.' 
Die Formen tati, tatira sind mit dem anaphorischen Pronomen (erweitert durch den Px3sg und Px2sg in determinierender Funktion - s. 2.1.3) identisch. Die Partikel taharia ist eine Entlehnung aus dem Russischen und geht auf das Spenderlexem manépb, manépя 'jetzt, nun' (Dal' 1882), eine dialektale Variante von ru. meneps 'jetzt, nun' zurück. ${ }^{25}$

Im Satz (21.5) wird tatirə anaphorisch verwendet: Ein vorher erwähntes Objekt ( ( "əmə 'mein Onkel') wird als Thema weitergeführt. Im Satz (21.8) funktioniert tatira textdeiktisch: Mit ihm wird auf die im vorigen Satz formulierte Proposition zurückverwiesen. Die übrigen Beispiele zeigen keine deiktische Funktion, hier wird die Bedeutung auch nicht auf der semantischen Ebene angesiedelt. Im Beispielsatz (21.14) könnte man noch einen anaphorischen Bezug vermuten, (21.11) schließt jedoch diese Deutung durch das Vorkommen des anaphorischen Personalpronomens siti eindeutig aus.

Die Partikeln sind im kognitiven Prozess der Sprachproduktion und des Sprachverstehens verankert. Sie erfüllen gleichzeitig mehrere kognitive Funktionen: Sprecherbezogen dienen sie der Gliederung des eigenen Gedankenganges, der Signalisierung des Anspruchs auf das Rederecht; hörerbezogen leisten sie die Gliederung der Informationsstruktur.

Wie kann man den oben dargestellten Prozess funktionalen Wandels beschreiben? Fernandez-Vest (2000) bezeichnet den Vorgang, in dem primär der Lokalisierung dienende sprachliche Mittel zur Thematisierung verwendet werden, als Grammatikalisierung. Die von ihr untersuchten Partikeln fi. tuota, totta, niin und lp. dat sind funktionale Äquivalente des Nganasanischen təti, tətirə. Gemeinsam sind diesen Partikeln nicht nur die Distribution und Funktionen (Gliederung der Informationsstruktur) im Text, sondern auch die ursprünglich deiktische Funktion. Gehört die Thema-Rhema-Gliederung in den Bereich der Grammatik? Oder sollen pragmatische Funktionen im Lexikon wiedergegeben werden? Wie schon erwähnt, ist die Funktion von təti als Topikmarker nicht grammatikalisiert. Für eine konsequente Begriffsverwendung scheint es adäquater, die beschriebene funktionale Erweiterung als Pragmatisierung zu betrachten.

${ }^{25}$ Mündliche Mitteilung von E. Helimski, für die ich mich an dieser Stelle bedanke. 


\section{Zusammenfassung: funktionale Verschiebungen des deiktischen Stammes to-}

Bei der vorliegenden Untersuchung der verschiedenen Verwendungsweisen der deiktischen Stämme im Nganasanischen und deren Ableitungen wurde zwischen deiktischen Ver-wendungen und nicht-deiktischen Verwendungen unterschieden. Das nganasanische anapho-rische Pronomen ta erscheint mit nicht-deiktischer Funktion in einer Vielzahl von Kontexten.

Dagegen finden sich kaum nicht-deiktische Verwendungen der proximalen und der distalen Demonstrativa (abgesehen von „Deixis ins Leere“). Dies hängt mit der unterschiedlichen Funktion der Demonstrativpronomina zusammen: Die anaphorische Bedeutung, der Rückbezug auf vorab Erwähntes, bleibt ein funktionaler Bestandteil der Komposita oder Ableitungen von tə- (z. B. in Konjunktionen, Modalpartikeln). Daher scheint unsere Annahme bestätigt, dass in der primären oder Kernfunktion des sprachlichen Zeichens das Spektrum seiner möglichen Entwicklungslinien vorkodiert ist.

Die verschiedenen funktionalen Wandlungen von $t$ ə- sind in der folgenden Abbildung zusammengefasst. Hypothetische Entwicklungslinien werden durch Kursivschrift gekennzeichnet. Der dunkelgraue Hintergrund trennt diejenigen Entwicklungen, die im Nganasanischen eingetreten sind, von den in der allgemeinen Grammatikalisierungsforschung (so z. B. bei Lehmann 1995) beschreibenen „klassischen“ Grammatikalisierungsketten. Auf diese Weise werden Charakteristika der nganasanischen Entwicklung deutlich (Tabelle 7).

Es ist interessant zu beobachten, welche typischen Grammatikalisierungsphänomene im Nganasanischen nicht stattgefunden haben: Das Nganasanische hat kein Relativpronomen und keine kataphorischen complementizer. Zum Ausdruck komplexer Propositionen hat das Nganasanische dagegen einen großen Bestand an Partizipialformen (Wagner-Nagy 2003, 177-82).

Des weiteren kennt das Nganasanische keinen bestimmten Artikel, wobei einige Verwendungen des anaphorischen Pronomens sowie des proximalen attributiven Demonstrativs auf eine Tendenz zur zunehmenden Markierung der Determination hindeuten. 


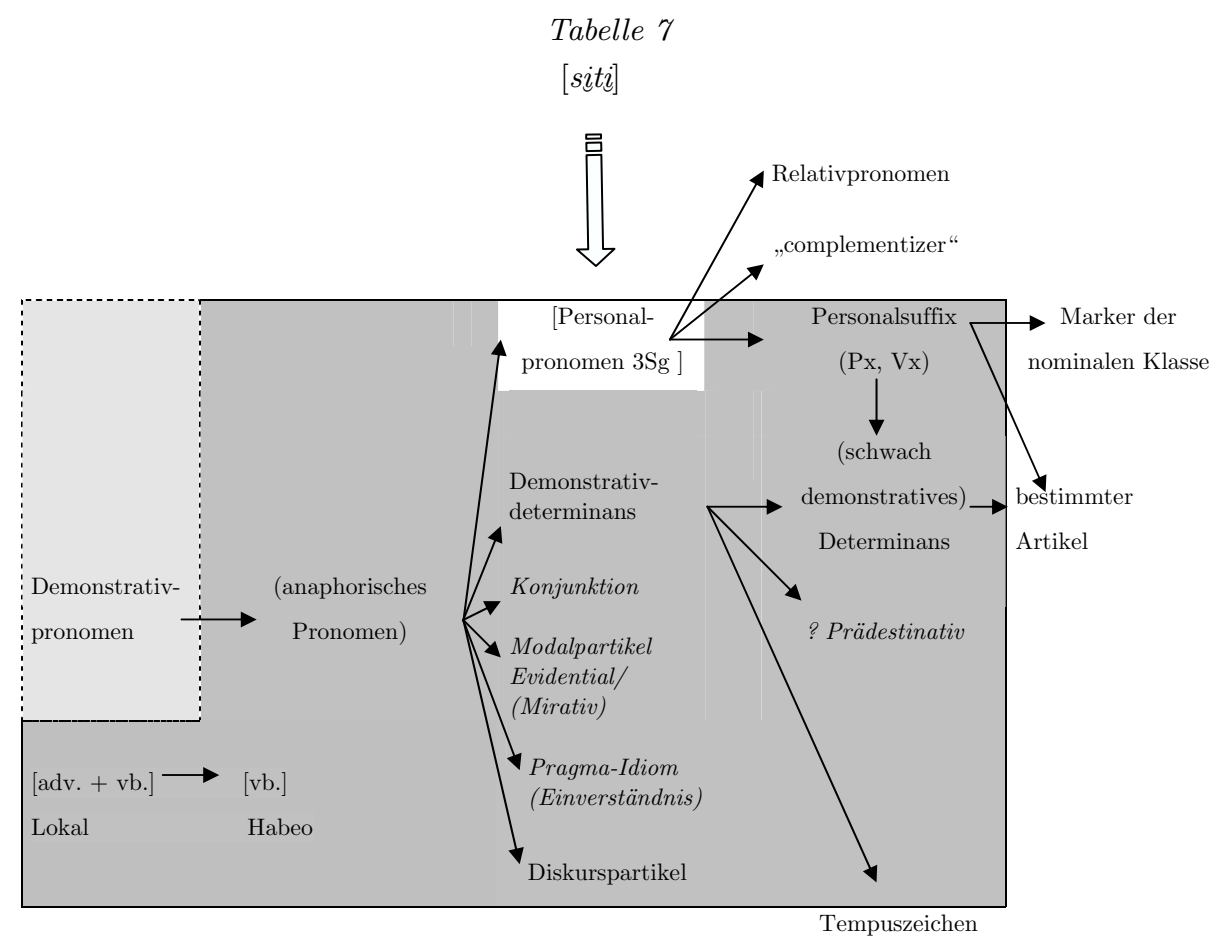

Abkürzungen

$\mathrm{A}=$ Akkusativ

abs $=$ absoluter Gebrauch attr $=$ attributiver Gebrauch

$\mathrm{du}=$ Dual

$\mathrm{El}=$ Elativ

evid $=$ Evidential
$\mathrm{G}=$ Genitiv

Ind $=$ Indikativ

Interr $=$ Interrogativ

Lat $=$ Lativ

mir $=$ Mirativ

$\mathrm{N}=$ Nominativ
Nsam. $=$ Nordsamojedisch präd $=$ Prädikat

Prol $=$ Prolativ

$\mathrm{PS}=$ Protosamojedisch

SK $=$ subjektive Konjugation

$\mathrm{U}=$ Uralisch

\section{Literatur}

Abondolo, Daniel (ed.) 1998. The Uralic languages. Routledge, London \& New York.

Abraham, Werner 1991. The grammaticalization of German modal particles. In: Elizabeth C. Traugott - Bernd Heine (eds): Approaches to grammaticalization. Vol II: Focus on types of grammatical markers. (Typological Studies in Language 19/1, 2), 331-80. John Benjamins, Amsterdam \& Philadelphia.

Castrén, Matthias Alexander 1854/1969. Grammatik der samojedischen Sprachen. Hrsg. von Anton Schiefner = Nordische Reisen und Forschungen 7. St. Petersburg \& Leipzig. 
Closs Traugott, Elizabeth 2002. Regularity in semantic change. Cambridge University Press, Cambridge.

Collinder, Björn 1965. An introduction to the Uralic languages. University of California Press, Berkeley.

Dal', Vladimir I. 1882. Толковый словарь живого великорусского языка. Государственное издателство иностранных и национальных словарей, С.Петербург \& Москва.

Diessel, Holger 1999a. Demonstratives. Form, function, and grammaticalization. John Benjamins, Amsterdam \& Philadelphia.

Diessel, Holger 1999b. The morphosyntax of demonstratives in synchrony and diachrony. In: Linguistic Typology $3: 1-49$.

Ehlich, Konrad 1982. Deiktische und phorische Prozeduren beim literarischen Erzählen. In: Eberhard Lämmert (ed.): Erzählforschung. Ein Symposium, 112-29. Metzler, Stuttgart.

Fernandez-Vest, Jocelyne M. M. 2000. Déixis, interaction, grammaticalisation: le cas des particules énonciatives en same du Nord et en finnois. In: Grammaticalisation aréale et sémantique cognitive: les langues fenniques et sames, OURAL - URAL nr 1. Paris.

Frajzyngier, Zygmunt 1996. On sources of demonstratives and anaphors. In: Barbara Fox (ed.): Studies in Anaphor. John Benjamins, Amsterdam \& Philadelphia.

Hajdú, Péter 1990. Einiges über Fürwörter. In: Linguistica Uralica 26:1-12.

Hajdú, Péter-Péter Domokos 1987. Die uralischen Sprachen und Literaturen. Buske, Hamburg.

Heine, Bernd 1992. Grammaticalization chains. In: Studies in Language 16:335-68.

Heine, Bernd - Tania Kuteva (eds) 2002. World lexicon of grammaticalization. Cambridge University Press, Cambridge.

Helimski, Eugene 1982. Древнейшие венгерско-самодийские языковые параллели. Наука, Москва.

Helimski, Eugene 1997. Die matorische Sprache. Wörterverzeichnis - Grundzüge der Grammatik - Sprachgeschichte (Studia Uralo-Altaica 41). SZTE Finnugor Tanszék \& MTA Nyelvtudományi Intézet, Szeged \& Budapest.

Helimski, Eugene 1998. Nganasan. In: Abondolo (1998, 480-515).

Helimski, Eugene 2003. Grammaticalization of abstracta in Samoyedic and Ugric languages: A collection of sample cases. Vortrag gehalten im Rahmen des Budapesti Uráli Mühely 4, Tischvorlage.

Himmelmann, Nikolaus 1997. Deiktikon, Artikel, Nominalphrase: Zur Emergenz syntaktischer Struktur. Niemeyer, Tübingen.

Itkonen, Erkki- Ulla-Maija Kulonen (eds) 1992-2000. Suomen sanojen alkuperä I-III. Suomalaissen Kirjallisuuden Seura, Helsinki.

Janhunen, Juha 1977. Samojedischer Wortschatz (Castrenianumin Toimitteita 17). Suomalais-ugrilainen Seura, Helsinki.

Janhunen, Juha 1981. Uralilaisen kantakielen sanastosta. In: Journal de la Société Finno-ougrienne $77: 219-69$.

Acta Linguistica Hungarica 51, 2004 
Janhunen, Juha 1989. Samojedin predestinatiivisen deklinaation alkuperästä. In: Journal de la Société Finno-ougrienne 82 : 298-301.

Janhunen, Juha 1998. Samoyedic. In: Abondolo (1998, 457-79).

Katzschmann, Michael 2003. Chrestomathia Nganasanica. Ms.

Kosterkina, Nadežda T. - Alexander Č. Momde- Tat'jana Ju. Ždanova 2001. Словарь нганасанско-русский и пусско-нганасанский. Просвещение, СанктПетербург.

Künnap, Ago 1987. Põhjasamojeedi predestinatiivsest deklinatsioonist. In: Journal de la Société Finno-ougrienne 81 : 209-220.

Labanauskas, K. I. 2001. Ня" дурымы" туобтугйся. Нганасанская фолклорная хрестоматия. The Nganasan Folklore Reader. Таимирский окружной центр народново творчества, Дудинка.

Lehmann, Christian 1995. Thoughts on grammaticalization (LINCOM Studies in Theoretical Linguistics 1). Lincom Europa, München \& Newcastle.

Lehtisalo, Toivo 1936. Über die primären ururalischen Ableitungssuffixe (Mémoires de la Société Finno-ougrienne 72). Suomalais-ugrilainen Seura, Helsinki.

Majtinskaja, Klara E. 1974. Основы финно-угорского языкознания. Вопросы происхождения и развития финно-угорских языков. Наука, Москва.

Mikola, Tibor 1965. A magyar tárgyrag eredetéhez. In: Nyelvtudományi Értekezések $46: 57-62$.

Mikola, Tibor 1988. Geschichte der samojedischen Sprachen. In: Sinor (1988, 219-63).

Paasonen, Heikki 1906. Über den ursprünglichen anlaut des finnischen demonstrativpronomens se. In: Finnisch-Ugrische Forschungen $6: 211-2$.

Ramat, Giacalone A.- Paul J. Hopper (eds) 1998. The limits of grammaticalization. John Benjamins, Amsterdam \& Philadelphia.

Redder, Angelika 1990. Sprachlicher Handel und Kommunikativer Absicht. „denn“ und „da“. Niemeyer, Tübingen.

Rédei, Károly (ed.) 1988-1991. Uralisches Etymologisches Wörterbuch I-III. Akadémiai Kiadó \& Harrasowitz, Budapest \& Wiesbaden.

Róna-Tas, András 1976. Az etimológia elméleti és módszertani kérdései a turkológiában. In: Nyelvtudományi Értekezések $89: 262-7$.

Sammallahti, Pekka 1988. Historical phonology of the Uralic languages. In: Sinor (1988, 478-554).

Sebestyén, Irén N. 1964. Két szamojéd visszaható névmás. In: Nyelvtudományi Közlemények 64 : 431-6.

Setälä, Emil Nestor 1902. Zur finnisch-ugrischen Lautlehre. In: Finnisch-Ugrische Forschungen $2: 268-9$.

Sinor, Denis (ed.) 1988. The Uralic languages. E. J. Brill, Leiden.

Tereščenko, Natal'ja Mitrofanovna 1979. Нганасанский язык. Наука, Ленинград.

Usenkova, E.V. 2003. Лексические и синтаксические средства выражения модальности в нганасанском языке. In: Linguistica Uralica 39:100-11.

Vértes, Edit 1967. Die ostjakischen Pronomina. Mouton, Bloomington \& The Hague. 
Wagner-Nagy, Beáta 2003. Chrestomathia Nganasanica (Studia-Uralo-Altaica Supplementum 10). SZTE Finnugor Tanszék \& MTA Nyelvtudományi Intézet, Szeged.

Willett, Thomas 1988. A cross-linguistic survey of the grammaticalization of evidentiality. In: Studies in Language 12:51-97.

Addresse der Verfasserin: Réka Zayzon

Finnisch-Ugrisches Seminar

Bogenallee 11

Hamburg

D-20144

Deutschland

Reka_Zayzon@public.uni-hamburg.de 